Die Abteilung Daten, Demographie und Qualität (DDQ) erstellt mit der Unterstützung der Arbeitsgruppe Qualität AGQ-FMH* Grundlagenpapiere zu verschiedenen Themen im Bereich Qualität, die in der Schweizerischen Ärztezeitung veröffentlicht werden. Die FMH nimmt auf der Basis der erarbeiteten Grundlagen Stellung zum Thema und gelangt über das Papier «Die Meinung der $\mathrm{FMH}$ » mit ihrer Position an die Öffentlichkeit. Nachfolgend werden das Grundlagenpapier sowie die Meinung der FMH zum Thema «Medikamentenverordnung» präsentiert.

\title{
Fehlerquelle Medikamentenverordnung
}

\author{
Varja A. Meyer-Nikolic, \\ Martina Hersperger, \\ Daniel Herren
}

\footnotetext{
* Mitglieder der AGQ-FMH (alphabetische Reihenfolge): Hélène Beutler, Christoph Bosshard (seit 28.6.2012), Karl Hampl, Catherine Heim, Daniel Herren (bis 7.6.2012), Martina Hersperger, Stefanie Hostettler, Esther Kraft, Monika Loy, Francesca Mainieri, Varja Meyer-Nikolic, Hans Anton Vogel, Jürg von Below, Adrian Wirthner
}

\section{Ausgangslage}

Medikamente sind notwendigerweise sowohl aus dem ärztlichen Praxisalltag wie auch aus dem Leben vieler Patienten nicht wegzudenken. Neben enormen medizinischen Möglichkeiten birgt die medikamentöse Behandlung aber auch ein beträchtliches Schadenspotential. Laut internationalen Studien gehören Medikationsfehler zu den häufigsten Fehlern im Gesundheitswesen überhaupt. Für die USA geht die Literatur davon aus, dass jährlich 5,2 Prozent aller hospitalisierten Patienten einen Medikationsfehler erleiden [1]. Im ambulanten Bereich ist gut ein Viertel der Patientinnen und Patienten von einem arzneimittel-assoziierten Problem betroffen. Davon sind 13 Prozent gravierend und mehr als ein Drittel vermeid- oder abschwächbar [2]. Die Anzahl vermeidbarer arzneimittel-assoziierter Probleme wird allein für die USA auf mindestens 1,5 Millionen Ereignisse pro Jahr geschätzt [3]. In erster Linie leidet der Patient darunter, aber auch die finanziellen Auswirkungen sind enorm. So unterstreicht auch die Weltgesundheitsorganisation (WHO) in ihrem 2008 publizierten Bericht «Research on Patient Safety» die Wichtigkeit von mehr Forschung und Interventionen in den Prozessen rund um die Arzneimitteltherapiesicherheit [4].

Dass auch in der Schweiz die Medikationssicherheit zu einem der wichtigsten Brennpunkte in der Patientensicherheit zählt, hat die Stiftung für Patientensicherheit 2006 in einer fundierten Expertenbefragung in Schweizer Spitälern bestätigt [5]. Schweizer Studien schätzen, dass mindestens 7,5 Prozent aller Patienten während ihres Spitalaufenthalts eine unerwünschte Medikamentenreaktion erleiden und/ oder von einem Medikationsfehler betroffen sind. Weiter zeigen sie auf, dass von allen Spitaleinweisungen in der Schweiz bis zu 7 Prozent auf ein arzneimittel-assoziiertes Problem zurückzuführen sind $[6,7,8]$. Schätzungen zufolge entstehen dadurch direkte Folgekosten von 70 bis 100 Millionen Franken [8]. Vor allem die hohe Spitaleinweisungsrate aufgrund von arzneimittel-assoziierten Problemen deutet darauf hin, dass die Medikationssicherheit auch im ambulanten Sektor nicht optimal gewährleistet ist. Genaue Angaben über die Häufigkeit von

\section{Zusammenfassung}

Medikationsfehler gehören zu den häufigsten Fehlern im Gesundheitswesen. Der Prozess der ärztlichen Medikamentenverordnung spielt dabei eine zentrale Rolle, denn genau in diesem ersten Prozessabschnitt treten sowohl im ambulanten als auch im stationären Sektor besonders häufig Fehler auf.

Dieses Papier zeigt die Komplexität der Medikamentenverordnung, die fehlerbegünstigenden Faktoren sowie mögliche Massnahmen für eine sicherere Verordnungspraxis auf.

ambulanten Medikationsfehlern fehlen für die Schweiz jedoch. Allerdings hat die Stiftung für $\mathrm{Pa}-$ tientensicherheit analog zum stationären Sektor auch bei ambulant tätigen Ärzten mittels einer Umfrage bei der Medikationssicherheit ein wichtiges Handlungsfeld eruiert [9]. Dies unterstützen auch internationale Studien, die aufzeigen, dass arzneimittel-assoziierte Probleme auch im ambulanten Setting häufig sind, obwohl viele davon vermieden werden könnten [10, 2].

Hinter dem Ziel, der richtigen Patientin das richtige Medikament zum richtigen Zeitpunkt in der richtigen Dosierung und Konzentration richtig zu verabreichen, steht ein komplexer und fehleranfälliger Prozess. Dieser reicht von der Verordnung über die Dokumentation, Dispensation und Verabreichung bis zum Monitoring. Jeder einzelne Schritt in diesem vielschichtigen und von verschiedenen Fachpersonen geprägten Prozess hat sein eigenes Gefahrenpotential. Der Schwerpunkt von diesem Papier soll auf der Medikamentenverordnung liegen - also demjenigen Prozessabschnitt, der in der Regel durch Ärztinnen und Ärzte ausgeführt wird. Für einen Überblick über den ganzen Medikationsprozess und seine Fehleranfälligkeit sind die Werke vom Institute of Medicine oft the National Acade- 
mies [3] und der American Pharmacists Association [11] empfehlenswert.

\section{Hot Spot Medikamentenverordnung}

Im Medikationsprozess nimmt die Verordnung von Medikamenten - eine primär ärztliche Handlung einen zentralen Stellenwert ein, da der gesamte Medikationsprozess auf einer korrekten Verordnung beruht. Laut Literatur entstehen viele der vermeidbaren Medikationsfehler jedoch genau in diesem ersten Prozessschritt, der die klinische Entscheidungsfindung, die Wahl des adäquaten Medikaments, die Festlegung der richtigen Dosierung, die Berücksichtigung der Medikamentencharakteristiken, die Dokumentation im Patientendossier sowie die schriftliche oder mündliche Medikamentenverordnung beinhaltet $[3,12]$. Einen Überblick über den Verordnungsprozess gibt die Abbildung 1. Typische Verordnungsfehler, die in diesen Prozessabschnitt gehören, sind beispielsweise eine fehlende

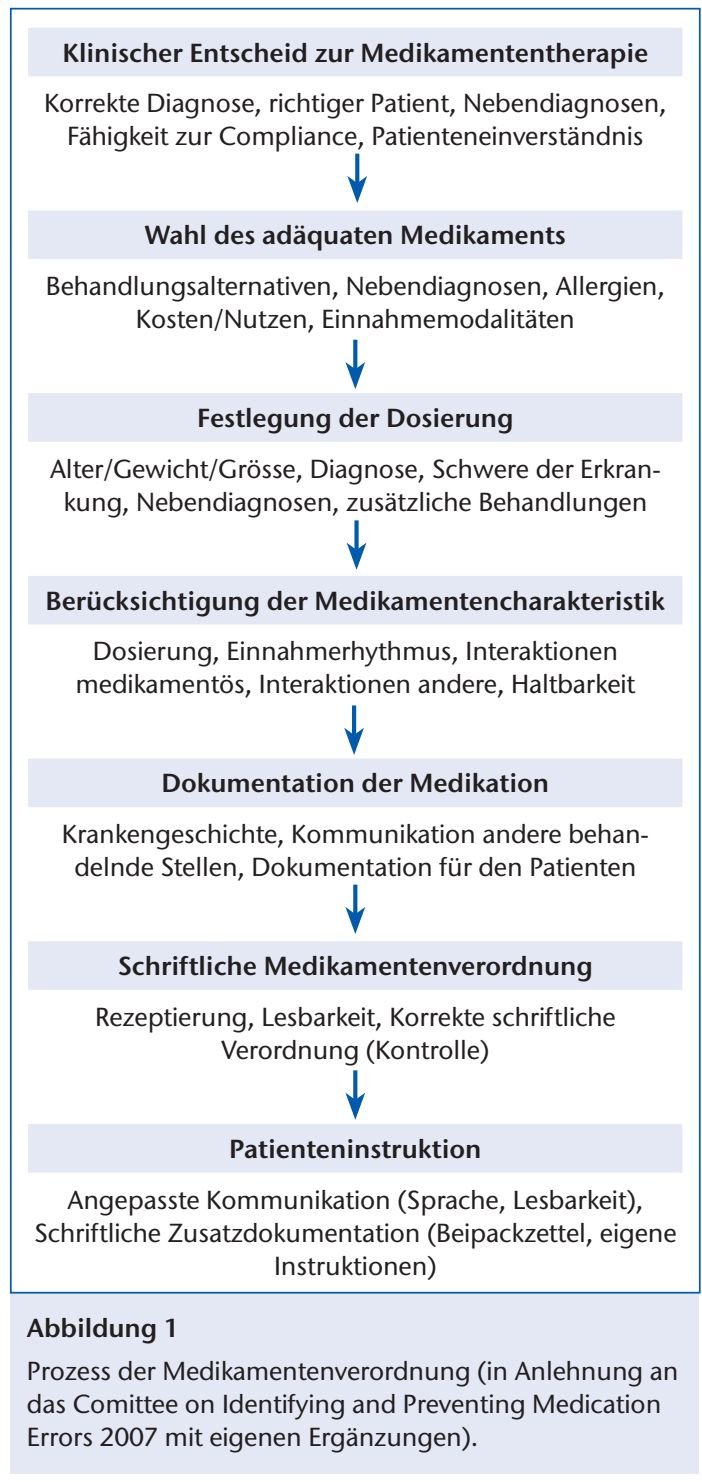

Dosierungsanpassung bei verminderter Nierenfunktion, die Verwendung von unkorrekten Abkürzungen oder die Missachtung von dokumentierten, patientenspezifischen Angaben wie z.B. das Vorliegen einer Allergie.

Obwohl nicht jeder Verordnungsfehler zu einem unerwünschten Ereignis führt, sind die Folgen von fehlerhaften Medikamentenverordnungen nicht zu unterschätzen. Sie können von unerwünschten Medikamenteninteraktionen über gefährliche Medikamenten-Krankheitsinteraktionen, allergischen Reaktionen, Überdosierungen bis hin zu Behandlungsmisserfolgen durch zu geringe Dosierungen führen. Dazu kommen fehlende Verordnungen trotz Indikation oder Verordnungen trotz fehlender Indikation beides Situationen, die ebenfalls unerwünschte Behandlungsergebnisse bewirken können [13]. Eine aktuelle Schweizer Studie zu Medikamenteninteraktionen zeigt zum Beispiel, dass 27 Prozent aller Verordnungen im stationären Sektor zu Medikamenteninteraktionen führen. 5 Prozent davon sind dabei klar als kontraindiziert zu kategorisieren [14].

\section{Was führt zu Verordnungsfehlern?}

So vielschichtig die Medikamentenverordnung in der Praxis ist, so komplex stellt sich auch die Ursachenanalyse dar, wenn es um die Entstehung von Verordnungsfehlern geht. Denn in den meisten Fällen führen nicht einzelne Faktoren zu medizinischen Fehlern in der Verordnungspraxis, sondern eine Kombination von mehreren fehlerbegünstigenden Umständen $[15,16]$.

Verschiedene Studien - vor allem aus dem stationären Bereich - haben sich mit den Ursachen von Verordnungsfehlern befasst [15, 17, 16]. Qualitative Interviews mit in Verordnungsfehler involvierten englischen Spitalärzten ergaben, dass dabei am häufigsten das Arbeitsumfeld (hohe Arbeitsbelastung, Arbeitsumgebung und Personalbesetzung), Teamfaktoren (Missverständnisse oder fehlende Kommunikation, unklare Verantwortlichkeiten, fehlende Supervision bei Assistenzärzten) sowie individuelle Faktoren (physisches und psychisches Unwohlsein wie Müdigkeit oder Stress, fehlendes Fachwissen) zu Verordnungsfehlern führen [15, 18] (Abb. 2).

Eine weitere wichtige Fehlerquelle bei handschriftlichen Verordnungen sind die mangelnde Lesbarkeit und Übertragungsfehler. Nur gerade zwei Prozent der untersuchten Verordnungen wurden als gut lesbar bewertet [17]. Die Folgen von unlesbaren oder missverständlichen Verordnungen können nebst schwerwiegenden medizinischen Konsequenzen auch zu unnötigen Arbeitsunterbrüchen führen, indem bei dem verordnenden Arzt nachgefragt werden muss. Solche Unterbrechungen der Arbeitsflüsse stellen wiederum weitere Fehlerquellen dar [19]. Wenn ergänzend institutionsbedingt eine Kultur existiert, die ein Nachfragen beispielsweise von Assistenzärzten oder Pflegefachpersonen bei ihren 


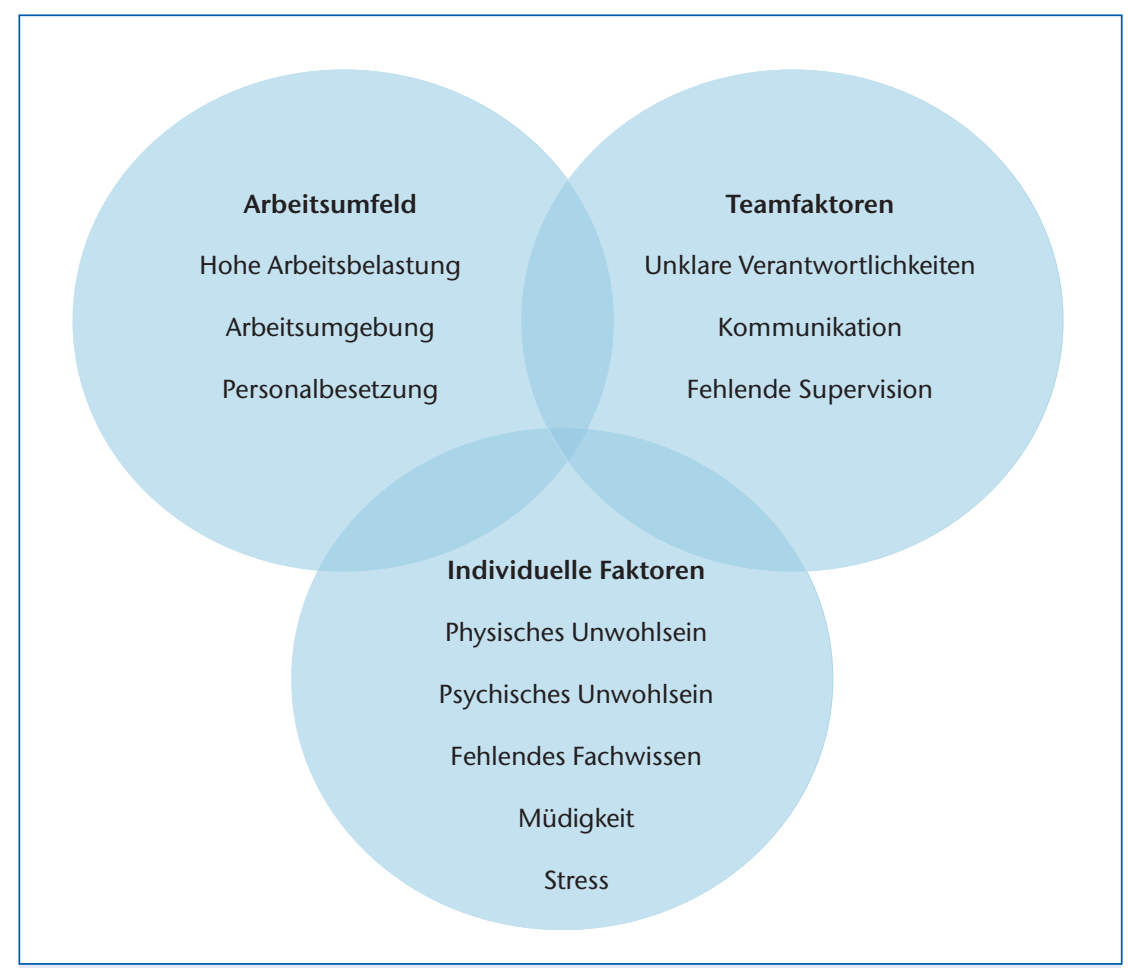

Abbildung 2

Begünstigende Faktoren für das Auftreten von Verordnungsfehlern im stationären Bereich aus Sicht des verschreibenden Arztes (in Anlehnung an Dean B. et al. (2002)).

Vorgesetzten be- oder verhindert, verschärft sich die Situation zusätzlich [15].

Explizite Studien, die sich vertieft mit den Ursachen für Verordnungsfehler im ambulanten Bereich auseinandersetzen, sind zum heutigen Zeitpunkt rar, wohl primär bedingt durch die Tatsache, dass die Datenerhebung deutlich schwieriger ist als im stationären Sektor. Grundsätzlich kann jedoch davon ausgegangen werden, dass die ähnlichen Faktoren wie im stationären Sektor auch im ambulanten Bereich zu Verordnungsfehlern führen. Was im ambulanten Sektor sicher eine besondere Herausforderung für das ärztliche Management von Medikamentenverordnungen darstellt, ist die Tatsache, dass häufig mehrere Ärzte für denselben Patienten Medikamente verordnen [20]. Vor allem lückenhafte Patientendokumentationen und eine mangelnde Kommunikation zwischen den verschiedenen an der Behandlung Beteiligten oder in der Arzt-Patienten-Beziehung sind diesbezüglich wichtige Gründe für Verordnungsfehler. Unwissenheit bezüglich patientenspezifischen Charakteristiken wie beispielsweise das Vorliegen von einer Schwangerschaft oder von Allergien, Labordaten, Alter und Gewicht, Diagnose oder selbstbezogene Medikamente oder Nahrungsergänzungsmittel sind ebenfalls Sicherheitsrisiken bei der Medikamentenverordnung, die in diese Kategorie gehören [3, 11].

Generell kann gesagt werden, dass die Kommunikation eine ganz zentrale Rolle bei der Verordnungs- sicherheit einnimmt. Ein unmissverständlicher Kommunikationsfluss ist nicht nur innerhalb eines behandelnden Teams oder in der Kommunikation mit dem Patienten elementar, sondern besonders auch an den Behandlungsschnittstellen zum Beispiel beim Spitaleintritt oder -austritt. Solche Schnittstellen in der Arzneimittelversorgung sind heikle Phasen und bedürfen besonderer Anstrengung beim Informationsfluss und der Vernetzung [21].

Eine weitere wesentliche und grundlegende Ursache für Fehler in der Medikamentenverordnung ist das fehlende Bewusstsein über die hohe Fehleranfälligkeit des Verordnungsprozesses an sich [16, 15]. Hinzu kommt die Tatsache, dass ein konstruktiver und offener Umgang mit Verordnungsfehlern - analog zum Umgang mit medizinischen Fehlern im Allgemeinen - häufig noch keine Selbstverständlichkeit darstellt. Dies verhindert die so wichtige Fehleranalyse und führt dazu, dass die gleichen Fehler immer wieder passieren.

\section{Massnahmen für eine sichere Verordnungspraxis}

Die Tatsache, dass Verordnungsfehlern ganz unterschiedliche Ursachen zugrunde liegen, bedingt auch für die Prävention von solchen Fehlern verschiedene Lösungsansätze. Allen Ansätzen zugrunde liegt sicher eine Verbesserung des angesprochenen bisher häufig fehlenden Bewusstseins der Verordnung als eigentliche Hochrisiko-Intervention. In den gleichen Lösungsansatz gehören eine bewusste Überprüfung der Verordnungsprozesse und die Analyse von Verordnungsfehlern dank einer etablierten Fehlerkultur. Es ist zentral, diesen Lösungsansätzen die angemessene Wichtigkeit zukommen zu lassen [15].

Wenn es am Anfang einer Verordnung darum geht, das richtige Medikament in der richtigen Dosierung zu bestimmen, sind Massnahmen wie die Sicherstellung eines adäquaten Zugangs zu Referenzmaterialien wie beispielsweise Guidelines oder Medikamenteninformationen wichtig [16]. Vor allem im stationären Bereich ist auch das Hinzuziehen von Pharmazeuten eine bedeutende Sicherheitsvorkehrung [15]. Zusätzlich existieren heutzutage verschiedene elektronische Verordnungshilfen. Studien konnten nachweisen, dass damit die Fehlerquote deutlich gesenkt werden kann [22]. Untersuchungen zeigen aber auch, dass solche Systeme auch neue Risiken für Fehler in der Medikamentenverordnung hervorrufen können, beispielsweise durch Fehler in der Handhabung solcher Tools [23]. Weiter sind viele elektronische Verordnungstools zu wenig auf patientenspezifische Eigenheiten ausgerichtet. Dies kann zu vielen falsch positiven Warnhinweisen führen und dadurch die Gefahr erhöhen, dass solchen Warnungen auch in komplexen Patientensituationen fälschlicherweise keine Beachtung geschenkt wird [24].

Wenn das richtige Medikament in der richtigen Dosierung bestimmt wurde, sind Massnahmen not- 
wendig, die eine korrekte Kommunikation der Verordnung gewährleisten. Auf mündliche Verordnungen sollte dabei möglichst verzichtet werden. Denn Missverständnisse sind vorprogrammiert. So wird zum Beispiel aus dem Medikament Cotrimazol schnell Clotrimazol oder umgekehrt [19]. Auch schriftliche Verordnungen sind nicht unproblematisch. Schon die Befolgung von einfachen Vorsichtsmassnahmen wie eine lesbare Handschrift oder die Einhaltung von Standards bei Abkürzungen kann zur Vorbeugung von Missverständnissen beitragen. Eine gute Übersicht zu dieser Problematik findet sich bei Cohen (2007) [19]. Einen wichtigen Beitrag leistet auch die elektronische Dokumentation, insbesondere die Verwendung von elektronischen Krankengeschichten, die unter anderem die Lesbarkeit gewährleisten [25].

Keine der genannten Massnahmen kann alle Verordnungsfehler reduzieren oder verhindern. Daher ist es relevant, dass die Thematik von verschiedenen Seiten her angegangen wird. Auf einen solchen ganzheitlichen Ansatz hat sich beispielsweise die Australian Commission on Safety and Quality in Health Care [26] oder das Bundesministerium für Gesundheit in Deutschland festgelegt. Seit 2007 wird in Deutschland ein umfangreicher Aktionsplan zur Verbesserung der Arzneimitteltherapiesicherheit für mehr Sicherheit im gesamten Prozess der Arzneimittelsicherheit umgesetzt. Zu den festgelegten Schritten gehören auch konkrete Massnahmen zur Verbesserung der Medikamentenverordnung [27].

\section{Was tut sich in der Schweiz?}

Von einer so umfassenden Strategie, wie sie in Deutschland umgesetzt wird, ist die Schweiz weit entfernt. Die hohe Prävalenz von arzneimittel-assoziierten Problemen prägt aber auch die nationale Gesundheitspolitik in der Schweiz. Neben verschiedenen aktuellen Vorstössen im National- und Ständerat [28] zur Verbesserung der Medikationssicherheit setzt auch die Qualitätsstrategie des Bundes im Schweizerischen Gesundheitswesen sowie deren Konkretisierungsbericht einen Schwerpunkt bei der Verbesserung der Medikationssicherheit. Als Ansätze für Interventionen nennen die Berichte u.a. die elektronische Verordnung, den pharmazeutischen Konsiliardienst zur individuellen Medikationsüberprüfung und die Medikamentenberatung bei Spitalaustritt $[29,30]$. So ist denn auch die elektronische Medikamentenverordnung einer der beiden prioritären Hauptprozesse bei der Umsetzung der nationalen Strategie eHealth Schweiz [33].

Neben den gesundheitspolitischen Ansätzen sind in der Schweiz auch einige vielversprechende Einzelinitiativen ergriffen worden. So engagiert sich beispielsweise die Stiftung für Patientensicherheit schon seit längerer Zeit in diversen Projekten für eine sicherere Arzneimitteltherapie [31]. Ein weiterer Ansatz sind die gemeinsamen Qualitätszirkel von
Ärzten und Apothekern beispielswiese im Kanton Freiburg. Diese möchten die Verordnungsqualiät sowie die Abläufe und Beziehungen zwischen Arzt und Apotheker kontinuierlich verbessern und gleichzeitig die Kosten unter Beibehaltung der Patientenorientierung des Arztes und des Apothekers senken [32].

\section{Schlussfolgerungen}

Medikamente $\mathrm{zu}$ verschreiben, ist eine komplexe und schwierige Aufgabe, doch die Häufigkeit, mit welcher Verordnungsfehler auftreten, ist deutlich zu hoch. Eine einzelne Massnahme, um die Verordnungspraxis zu verbessern, reicht alleine nicht aus. Ein multidimensionaler Ansatz ist dringend notwendig. Hierzu gehören auch die Förderung der Forschung zur Entwicklung und Implementierung von praxistauglichen Instrumenten sowie die Koordination der verschiedenen Ansätze und die Übertragung von bereits erprobten Massnahmen. Besonders im ambulanten Bereich wären auch Studien zur Ermittlung der Ursachen von Verordnungsfehlern wichtig.

Weil der gesamte Medikationsprozess auf einer korrekten Verordnung beruht, ist es zentral, in diesen ersten Prozessabschnitt nachhaltig zu investieren. Hier ist die Ärzteschaft besonders gefordert, da die Medikamentenverordnung primär eine ärztliche Tätigkeit darstellt. Da die Ursachen für Verordnungsfehler jedoch häufig auf der Systemebene anzusiedeln sind, ist eine gemeinsame Lösungsstrategie von allen beteiligten Berufsgruppen und Institutionen zwingend notwendig.

Das komplexe Themenfeld verunmöglicht es, allen Ursachen für und Lösungsansätzen gegen Verordnungsfehler gerecht zu werden. Daher erhebt dieses Papier auch keinen Anspruch auf Vollständigkeit. Ein erster wichtiger Schritt ist jedoch die Schärfung des Bewusstseins für die Verordnung als mögliche Hochrisiko-Intervention - wozu hoffentlich auch das vorliegende Grundlagenpapier seinen Beitrag leisten wird.

\section{Literatur}

1 Bond CA, Raehl CL, Franke T. Clinical Pharmacy Services, Hospital Pharmacy Staffing, and Medication Errors in the United States Hospitals. Pharmacotherapy. 2002;22(2):1344-7.

2 Gandhi TK, Weingart SN, Borus J, Seger AC, Peterson J, et al. Adverse Drug Events in Ambulatory Care. The New England Journal of Medicine. 2003;248(16):155664.

3 Comittee on Identifying and Preventing Medication Errors, Institute of Medicine of the National Academies. Preventing Medication Errors. Washington: The National Academies Press; 2007.

4 World Health Organisation WHO/The Research Priority Setting Working Group of the World Alliance for Patient Safety. Summary of the evidence on patient safety: Implications for Research. Online in Internet: http://whqlibdoc.who.int/publica- 
tions/2008/9789241596541_eng.pdf; 2008. [Stand: 17.2.2012].

5 Frank O, Hochreutener MA. Problemfelder (Hot-Spots) in der Patientensicherheit. Schweiz Ärztezeitung. 2008;89(24):1079-82.

6 Hardmeier B, Braunschweig S, Cavallaro M, Roos M, Pauli-Magnus, et al. Adverse drug events caused by medication errors in medical inpatients. SwissMedWkly. 2004;134(45/46):664-70.

7 Fattinger K, Roos M, Vergères P, Holenstein C, Kind B, et al. Epidemiology of drug exposure and adverse drug reactions in two swiss departments of internal medicine. British Journal of Clinical Pharmacology. 2000;49(2):158-67.

8 Lepori V, Perren A, Marone C. Adverse internal medicine drug effects at hospital admission. Schweiz Med Wochenschrift. 1999;129(24):915-22.

9 Gehrin K, Schwappach D. Patientensicherheit in der Grundversorgung - erste Daten aus der Schweiz. Schweiz Ärztezeitung. 2011;92(39):1486-7.

10 Aagaard Thomsen, L, Winterstein AG, Søndergaard B, Stig Haugbølle L, Melander A. Systematic Review oft he Incidence and Characteristics of Preventable Adverse Drug Events in Ambulatory Care. The Annals of Pharmacotherapy. 2007;41:1411-26.

11 Cohen M. Causes of Medication Errors. In: Cohen M (eds.). Medication Errors. Washington: American Pharmacists Association; 2007. p. 55-66.

12 Bates DW, Cullen DJ, Laird N, Petersen LA, Small SD et al. Incidence of adverse drug events and potential adverse drug events: implications for prevention. JAMA. 1995;274:29-34.

13 Wessell AM, Litvin C, Jenkins RG, Nietert PJ, Nemeth LS, et al. Medication prescribing and monitoring errors in primary care: a report from the Practice Partner Research Network. Quality and Safety in Health Care BMJ. 2010;19(e21). Online in Internet: http:// qualitysafety.bmj.com/content/19/5/1.39.short [18.10.2012].

14 Oertle M. Frequency and nature of drug-drug interactions in a Swiss primary and secondary acute care hospital. SwissMedWkly. 2012;142:w13522.

15 Dean B, Schachter M, Vincent C, Barber N. Causes of prescribing errors in hospital inpatients: a prospective study. The Lancet. 2002;359:1373-8.

16 Coombes ID, Stowasser DA, Coombes JA, Mitchell C. Why do interns make prescribing errors? A qualitative study. Medical Journal of Australia. 2008;188(2):89-94

17 Hartel M, Staub LP, Röder C, Eggli S. High incidence of medication documentation errors in a Swiss university hospital due to the handwritten prescription process. BMC Health Services Research. 2011;11:199.

18 Zum Zusammenhang zwischen dem ärztlichen Wohlbefinden und der Behandlungsqualität hat die Abteilung Daten, Demographie und Qualität (DDQ) der FMH ein eigenes Grundlagenpapier publiziert. Siehe dazu: Hostettler, Hersperger, Herren. Ärztliches Wohlbefinden beeinflusst die Behandlungsqualität. Schweiz Ärztezeitung. 2012;93(18):655-9.

19 Cohen M. Causes of Medication Errors. In: Cohen M. (eds.). Medication Errors. Washington: American Pharmacists Association; 2007. p. 175-203.
20 Rundall TG, Hsu J, Elston Lafata J, Fung V, Paez KA, et al. Prescribing Safety in Ambulatory Care: Physician Perspectives. In: Henriksen K, et al. (eds.). Advances in Patient Safety: From Research to Implementation (Vol. 1: Research Findings). Rockville (MD): Agency for Healthcare Research and Quality (US); 2005. p. 161-71.

21 Hersberger K, Petitjean-Wiesner C. Arzneimittelversorgung in der Perihospitalphase: Die Schnittstelle Spital/ Apotheke/Hausarzt. Managed Care. 2004;1:10-2.

22 Siehe z.B. Kaushal R, Kern LM, Barrón Y, Quaresimo J, Abramson EL. Electronic Prescribing Improves Medication Safety in Community-Based Office Practices. Journal of General Internal Medicine. 2010;25(6):530-6.

23 Siehe z. B. Redwood S, Rajakumar A, Hodson J, Coleman JJ. Does the implementation of an electronic prescribing system crate unintended medication errors? A study of the sociotechnical context through the analysis of reported medication incidents. BMC Medical Informatics and Decision Making. 2011;11:29. Online: www.biomedcentral.com/1472-6947/11/29 [Stand: 22.2.2012]

24 Taylor LK, Kawasumi Y, Bartlett G, Tamblyn R. Inappropriate Prescribing Practices: The Challenge and Opportunity for Patient Safety. Healthcare Quarterly. 2005; Vol. 8, Special Issue:81-5.

25 Schade CP, Sullivan FM, De Lusignan S, Madeley J. e-Prescribing, Efficiency, Quality: Lessons from Computerization of UK Familiy Practice. Journal of the American Medical Informatics Association. 2006;13:470-5.

26 Australian Commission on Safety and Quality in Health Care: Medication Safety. Online in Internet: www.safetyandquality.gov.au/our-work/medicationsafety/ [18.10.2012]

27 Bundesministerium für Gesundheit: Aktionsplan zur Verbesserung der Arzneimitteltherapiesicherheit in Deutschland. Online in Internet: www.ap-amts.de/ [Stand: 17.2.2012]

28 Siehe z. B. www.parlament.ch/d/suche/seiten/ geschaefte.aspx?gesch_id=20103093 [Stand 6.3.2012]

29 Bundesamt für Gesundheit. Qualitätsstrategie des Bundes im Schweizerischen Gesundheitswesen. Online in Internet: www.bag.admin.ch/themen/ krankenversicherung/00300/00304/index. html?lang=de; 2009. [Stand 23.11.2011].

30 Bundesamt für Gesundheit. Bericht an den Bundesrat zur Konkretisierung der Qualitätsstrategie des Bundes im Schweizerischen Gesundheitswesen. Online in Internet: www.bag.admin.ch/themen/krankenversicherung/00300/00304/index.html?lang=de; 2011. [Stand: 23.11.2011].

31 Siehe www.patientensicherheit.ch. [Stand: 17.2.2012].

32 Nyffeler R. Qualitätszirkel zur Medikamentenverschreibung - eine Antwort auf die Kostenentwicklung im Gesundheitswesen. Managed Care. 2002;(2):23-6.

33 Siehe Website von eHealth Suisse: www.e-healthsuisse.ch/umsetzung/00146/00148/index. html?lang=de [Stand: 4.7.2012]. 